\title{
Altretamine Regimen
}

National Cancer Institute

\section{Source}

National Cancer Institute. Altretamine Regimen. NCI Thesaurus. Code C159987.

A chemotherapy regimen consisting of altretamine that may be used in the treatment of ovarian, fallopian tube, and primary peritoneal cancers. 